Original Research Paper

\title{
q-Deformation of Transonic Gas Equation
}

\author{
${ }^{1,2}$ Sami H. Altoum \\ ${ }^{1}$ Academy of Engineering Science, Sudan \\ ${ }^{2}$ Department of Mathematics, AL-Qunfudhah University College, Umm Al-Qura University, Saudi Arabia
}

Article history

Received: 23-09-2017

Revised: 23-11-2017

Accepted: 24-4-2018

Email: samialtoum $@$ hotmail.com

\begin{abstract}
In this study, the transonic gas equation will be considered. For $q \in(0,1)$, q-deformation of transonic gas equation ( $q$-transonic) are studied, we use q- derivative (or Jackson derivative) to solve transonic gas equation.
\end{abstract}

Keyword: Transonic Gas Equation, q-Deformation, q-Derivative

\section{Introduction}

In real complex and functional analysis, derivatives are generalized to functions of several real or complex variables and functions between topological vector spaces. An important case is the variational derivative in the calculus of variations. Repeated application of differentiation leads to derivatives of higher order and differential operators. The derivative is often met for the first time as an operation on a single real function of a single real variable. One of the simplest settings for generalizations is to vector valued functions of several variables (most often the domain forms a vector space as well). This is the field of multivariable calculus. In one-variable calculus, we say that a function $f: \mathbb{R} \rightarrow \mathbb{R}$ is differentiable at a point $x$ if the limit $\lim _{h \rightarrow 0} \frac{f(x+h)-f(x)}{h}$ exists. Roughly speaking, in mathematics, specifically in the areas of combinatorics and special functions, a $q$-analog of a theorem, identity or expression is a generalization involving a new parameter $q$ that returns the original theorem, identity or expression in the limit as $q \rightarrow 1$ (this limit is often formal, as $q$ is often discrete-valued). Typically, mathematicians are interested in $q$ analog that arise naturally, rather than in arbitrarily contriving $q$-analog of known results. The earliest $q$ analog studied in detail is the basic hypergeometric series, which was introduced in the 19th century. $q$ analog find applications in a number of areas, including the study of fractals and multi-fractal measures and expressions for the entropy of chaotic dynamical systems. The relationship to fractals and dynamical systems results from the fact that many fractal patterns have the symmetries of Fuchsian groupssee (Accardi and Boukas, 2006; Accardi et al., 2006; Ettaieb et al., 2014; Leeuwen and Maassen, 1995; Hida, 1993). The connection passes through hyperbolic geometry and ergodic theory, where the elliptic integrals and modular formsplay a prominent role; the $q$-series themselves are closely related to elliptic integrals. $q$-analogs also appear in the study of quantum groups and in $q$ deformed superalgebras. The connection here is similar, in that much of string theory is set in the language of Riemann surfaces, resulting in connections to elliptic curves, which in turn relate to $q$-series. There are two main groups of $q$-analogs, the "classical" $q$ analogs, with beginnings in the work of Leonhard Euler and extended by F.H. Jackson and others. qanalogs are often found in physical applications, especially exact solutions of many-body problems. In such cases, the $q \rightarrow 1$ limit usually corresponds to relatively simple dynamics, e.g., without nonlinear interactions, while $q<1$ gives insight into the complex nonlinear regime with feedbacks. An example from atomic physics is the model of molecular condensate creation from an ultra-cold fermionic atomic gas during a sweep of an external magnetic field through the Fischbach resonance. This process is described by a model with a $q$-deformed version of the SU(2) algebra of operators (The special unitary group $\mathrm{SU}(n)$ is a real Lie group though not a complex Lie group) (Accardi and Boukas, 2009; Bangerezako, 2004; Bożejko and Speicher, 1991; Ettaieb et al., 2012; Jackson, 1910; Mason, 1915) and its solution is described by $q$-deformed exponential and binomial distributions. This article is organized as follows: In section 2 we state the main problem transonic gas equation, in section 3 introduced q-derivative, in section 4 introduced some q-calculus definitions related to q-deformation and Jackson derivative, in section 5 the classical solution of transonic gas equation presented. Finally used to q-deformation to deduce a solution of transonic gas equation. 


\section{Transonic Gas Equation}

Consider the transonic gas equation:

$u_{x} u_{x x}+u_{y y}=0$

The study of gas dynamics is often associated with the flight of modern high-speed aircraft and atmospheric reentry of space-exploration vehicles; however, its origins lie with simpler machines. At the beginning of the 19th century, investigation into the behavior of fired bullets led to improvement in the accuracy and capabilities of guns and artillery. As the century progressed, inventors such as Gustaf de Laval advanced the field, while researchers such as Ernst Mach sought to understand the physical phenomenon involved through experimentation the model equation gas equation. In classical solution of transonic gas flow the solution take a system of equations but the number of equations is less the number of unknowns parameters, for this reason we cannot have a unique solution for our differential equation. This leads to an open question, what can we do in our case or how can we change completely the PDEs in order to get a solvable system with unique solution. So in this study we solve the transonic gas equation using q-deformation.

\section{q-Derivative}

Here is a nice diversion for anyone who knows what the derivative of a simple function is $f(x)$. The modern theory of differential and integral calculus began in the 20th century with the works of Newton and Leibniz. As it is well known, the derivative of a function $f(x)$ with respect to the variable $\mathrm{x}$ is by definition:

$$
f^{\prime}(x)=\lim _{h \rightarrow 0} \frac{f(x+h)-f(x)}{h} .
$$

Now, let us consider the following expression:

$$
f^{\prime}(x)=\lim _{q \rightarrow 1} \frac{f(q x)-f(x)}{q x-x} .
$$

Of course, this is not valid when $q=1$ or $x=0$ but otherwise this alternative formula is equivalent to the usual derivative. You can convince yourself by writing $\frac{f(x+(q-1) x)-f(x)}{(q-1) x}$, the term $(q-1) x$ playing the role of $h$.

At the beginning of the 20th century, F.H. Jackson studied this modified derivative and many of its consequences. The key concept is the q-derivative operator defined as follows when $0<q<1$ :

$$
\left(D_{q} f\right)(x)=\lim \frac{f(q x)-f(x)}{q x-x},
$$

this q-derivative can be applied to functions not containing 0 in their domain of definition. Then it reduces to the ordinary derivative when $q$ goes to 1 :

$$
\lim _{q \rightarrow 1}\left(D_{q} f\right)(x)=f^{\prime}(x)
$$

\section{Example}

Compute the q-derivative of $x^{2}+2 x+1$ :

$$
\begin{aligned}
& D_{q}\left(x^{2}+2 x+1\right)=\frac{\left[(q x)^{2}+2(q x)+1\right]-\left[x^{2}+2 x+1\right]}{q x-x} \\
& =(1-q) x+2 .
\end{aligned}
$$

One can easily check that the q-derivative operator is linear:

$$
\begin{aligned}
& D_{q}(f+g)=D_{q} f+D_{q} g \\
& D_{q}(\lambda f)=\lambda D_{q} f,
\end{aligned}
$$

the product rule is slightly modified but it approaches the usual product rule when $q$ goes to one:

$$
\left(D_{q}(f g)\right)(x)=f(q x)\left(D_{q} g\right)(x)+\left(D_{q} f\right)(x) g(x) .
$$

\section{q-Calculus}

We recall some basic notations used in q-calculus (Abdi, 1962; Accardi and Boukas, 2007; Adams, 1929; Altoum et al., 2047; Ettaieb et al., 2016; Hida, 1993; Yuri et al., 1998). The natural number $n$ has the following $q$ deformation:

$$
[n]_{q}:=1+q+q^{2}+\cdots+q^{n-1}, \text { with }[0]_{q}=0 .
$$

Occasionally, we shall write $[\infty]_{q}$ for the limit of these numbers:

$$
\frac{1}{1-q}
$$

The $q$ factorials and $q$ binomial coefficients are defined naturally as:

$$
[n]_{q} !=[1]_{q} \cdot[2]_{q} \cdot[3]_{q} \ldots[n]_{q}, \text { with }[0]_{q}:=0 .
$$

Recall that from (Yuri et al., 1998), for $q \in(0,1)$ relation $a a^{*}-q a^{*} a=1$ (q-Deformation of the Square 
White Noise Lie Algebra) admits, up to unitary equivalence, a unique non-trivial bounded irreducible representation given on the canonical basis:

$$
\left\{e_{n} \mid n \in \mathbb{N}\right\} \text { of } l^{2}(\mathbb{N})
$$

Defined using the following equations:

- $a^{*} e_{n}=e_{n+1}$

- $a e_{n}=[n]_{q} e_{n-1}$

- $\left\langle e_{n}, e_{m}\right\rangle=\delta_{n, m}[n]_{q}$ !

For $q \in(0,1)$ and analytic for $f: \mathbb{C} \rightarrow \mathbb{C}$ define operators $Z$ and $D_{q}$ as follows (Ettaieb et al., 2016; Hida, 1993; Yuri et al., 1998):

$$
\begin{aligned}
& (Z f)(z):=z f(z), \\
& \left(D_{q} f\right)(z)=\left\{\begin{array}{c}
\frac{f(z)-f(q z)}{z(1-q)}, z \neq 0 \\
f^{\prime}(0)
\end{array}\right.
\end{aligned}
$$

The operator $D_{q}$ has the following properties:

- $\lim _{q \rightarrow 1}\left(D_{q} f\right)(x)=f^{\prime}(x)$

- $D_{q}\left(z^{n}\right)=\frac{1-q^{n}}{1-q} z^{n-1}=[n]_{q} z^{n-1}$

- $\quad D_{q}\left(f(z) g(z)=\left(D_{q} f\right)(z) g(z)+f(q z)\left(D_{q} g\right)(z)\right.$

- $D_{q} \frac{f(z)}{g(z)}=\frac{\left(D_{q} f\right)(z) g(z)-f(z)\left(D_{q} g\right)(z)}{g(z) g(q z)}$

We can apply the Taylor formula to the definition of $\left(D_{q} f\right)(z)$ to get:

$$
\left(D_{q} f\right)(z)=\sum_{k=0}^{\infty} \frac{(q-1)^{k}}{(k+1) !} x^{k} f^{(k+1)}(x) .
$$

A $q$-analog of the Taylor expansion of a function around zero as follows:

$$
f(z)=\sum_{n=0}^{\infty} f^{n}(0) \frac{(z)^{n}}{(n) !}=\sum_{n=0}^{\infty}\left(D_{q}^{n} f\right)(0) \frac{z^{n}}{[n] !} .
$$

\section{Classical Solution of Transonic Gas Equation (Titov, 1988)}

The exact solution of the transonic gas dynamics given by the Equation 1 is as follows.

Suppose $u(x, t)$ is the solution of the Equation 1. Then the function:

$$
u_{1}=c_{1}^{-3} c_{2}^{2} u\left(c_{1} x+c_{3}, c_{2} y+c_{4}\right)+c_{5} y+c_{6},
$$

is also a solution, where $c_{1}, c_{2}, \ldots, c_{6}$ are arbitrary constants. Solution can be investigated are the following:

$$
\begin{aligned}
& u(x, t)=c_{1} x y+c_{2} x+c_{3} y+c_{4} \\
& u(x, t)=\frac{a^{2} c_{1}^{3}}{39}(y+A)^{13}+\frac{2}{3} a(y+A)^{8}(x+B) \\
& +3 c_{1}(y+A)^{3}(x+B)^{2}-\frac{(x+B)^{3}}{3 a(y+A)^{2}} \\
& u(x, t)=\frac{\left(x+c_{1}\right)^{2}}{3 a\left(x+c_{1}\right)^{3}}+c_{3} y+c_{4} \\
& u(x, t)=-a A^{3} y^{2}-\frac{B^{2}}{a A^{2}} x+c_{1}+c_{2} \pm \frac{4}{3}\left(A x+B y+c_{3}\right)^{\frac{3}{2}} \\
& u(x, t)=\frac{1}{3}(A y+B)\left(2 c_{1} x+c_{2}\right)^{\frac{3}{2}}-\frac{a c_{1}^{3}}{12 A^{2}}(A y+B)^{4}+c_{3} y+c_{4} \\
& u(x, t)=-\frac{9 a A^{2}}{y+c_{1}}+4 A\left(\frac{x+c_{2}}{y+c_{1}}\right)^{\frac{3}{2}}-\frac{\left(x+c_{2}\right)^{3}}{3 a\left(y+c_{1}\right)^{2}}+c_{3} y+c_{4} \\
& u(x, t)=-\frac{3}{7} a A^{2}\left(y+c_{1}\right)^{7}+4 A\left(x+c_{2}\right)^{\frac{3}{2}}\left(y+c_{1}\right)^{\frac{5}{2}} \\
& -\frac{\left(x+c_{2}\right)^{3}}{3 a\left(y+c_{1}\right)^{2}}+c_{3} y+c_{4}
\end{aligned}
$$

where, $A, B, C_{1}, \ldots, C_{4}$, are arbitrary constant (the first solution is degenerate).

There are solutions of the following forms:

- self-similar solution:

$$
z=x y^{k}, u(x, t)=y^{-3 k-2} u(z), z=x y^{k}
$$

where, $k$ is any arbitrary constants.

- Generalized separable solution as:

$$
\begin{aligned}
& u(x, y)=\phi_{1}(y)+\phi_{2}(y) x^{\frac{3}{2}}+\phi_{3}(y) x^{3} \\
& u(x, t)=\psi_{1}(y)+\psi_{2}(y) x+\psi_{3}(y) x^{2}+\psi_{4}(y) x^{3} \\
& u(x, t)=\psi_{1}(y) \phi(x)+\psi_{2}(y)
\end{aligned}
$$

\section{Analogous of Transonic Gas Equation}

As an $q$-analogous of (1), we introduce $q$-transonic gas equation as follows:

$\left(D_{q, x} u\right)\left(D_{q, x}^{2}\right)+\left(D_{q, y}^{2}\right)=0$,

where:

$$
D_{q} f(r)=\left\{\begin{array}{c}
\frac{f(r)-f(q r)}{r-q r} r \neq 0, \quad 0<q<1, \\
f^{\prime}(0)
\end{array}\right.
$$


then:

$$
D_{q, x} f\left(z_{1}, z_{2}\right)=\left\{\begin{array}{c}
\frac{f\left(z_{1}, z_{2}\right)-f\left(q z_{1}, z_{2}\right)}{z_{1}-q z_{1}}, \quad i f z_{1} \neq 0, \\
\partial_{x} f\left(0, z_{2}\right)
\end{array}\right.
$$

and:

$$
D_{q, y} f\left(z_{1}, z_{2}\right)=\left\{\begin{array}{c}
\frac{f\left(z_{1}, z_{2}\right)-f\left(z_{1}, q z_{2}\right)}{z_{2}-q z_{2}}, \\
\partial_{y} f\left(z_{1}, 0\right)
\end{array}\right.
$$

and:

$$
\begin{aligned}
& D_{q, x x} f\left(z_{1}, z_{2}\right) \\
& =\left\{\begin{array}{c}
\frac{f\left(z_{1}, z_{2}\right)-2 f\left(q z_{1}, z_{2}\right)-f\left(q^{2} z_{1}, z_{2}\right)}{z_{1}^{2}-(1-q)^{2}}, \text { if } z_{1} \neq 0, \\
\partial_{x x} f\left(0, z_{2}\right)
\end{array}\right.
\end{aligned}
$$

and:

$$
\begin{aligned}
& D_{q, y y} f\left(z_{1}, z_{2}\right) \\
& =\left\{\begin{array}{c}
\frac{f\left(z_{1}, z_{2}\right)-2 f\left(z_{1}, q z_{2}\right)+f\left(z_{1}, q^{2} z_{2}\right)}{z_{2}^{2}-(1-q)^{2}}, \text { if } z_{2} \neq 0 . \\
\partial_{y y} f(z, 0)
\end{array}\right.
\end{aligned}
$$

Now let:

$$
\begin{aligned}
& D_{q, x y} f\left(z_{1}, z_{2}\right)=D_{q, x}\left(D_{q, y} F\left(z_{1}, z_{2}\right)\right) \\
& =\left\{\begin{array}{c}
\frac{F\left(z_{1}, z_{2}\right)-F\left(q z_{1}, z_{2}\right)}{z_{1}-(1-q)}, \text { if } z_{1} \neq 0 \\
\partial_{x} f\left(0, z_{2}\right)
\end{array}\right. \\
& =\left\{\begin{array}{c}
\frac{f\left(z_{1}, z_{2}\right)-f\left(z_{1}, q z_{2}\right)-f\left(q z_{1}, z_{2}\right)+f\left(q z_{1}, q z_{2}\right)}{z_{1} z_{2}(1-q)^{2}}, z_{1} \neq 0, z_{2} \neq 0 \\
\frac{\partial_{y} f(z, 0)-\partial_{y} f\left(q z_{1}, 0\right)}{z_{1}(1-q)}, z_{1} \neq 0, z_{2}=0 \\
\frac{\partial_{x} f\left(0, z_{2}\right)-\partial_{x} f\left(0, q z_{2}\right)}{z_{1}(1-q)}, \quad z_{1}=0, z_{2} \neq 0 \\
\partial_{x y} f(0,0),
\end{array}\right.
\end{aligned}
$$

where, $\left(z_{1} \neq 0 \Lambda z_{2} \neq 0\right) \Rightarrow z_{1}=0 \mathrm{~V} z_{2}=0$.

Let:

$$
D_{q, x} f\left(z_{1}, z_{2}\right)=g\left(z_{1}, z_{2}\right)
$$

then:

$$
\begin{aligned}
& D_{q, y x} f\left(z_{1}, z_{2}\right)=D_{q, y}\left(D_{q, x} F\left(z_{1}, z_{2}\right)\right) \\
& =D_{q, y} g\left(z_{1}, z_{2}\right) \\
& =\left\{\begin{array}{c}
\frac{D_{q, x} f\left(z_{1}, z_{2}\right)-D_{q, x} f\left(z_{1}, q z_{2}\right)}{z_{2}(1-q)}, \quad z_{2} \neq 0 \\
\partial_{y} D_{q, x} f\left(z_{1}, 0\right)
\end{array}\right. \\
& \int \frac{f\left(z_{1}, z_{2}\right)-f\left(q z_{1}, z_{2}\right)-f\left(z_{1}, q z_{2}\right)+f\left(q z_{1}, q z_{2}\right)}{z_{1} z_{2}(1-q)^{2}}, z_{1} \neq 0, z_{2} \neq 0
\end{aligned}
$$

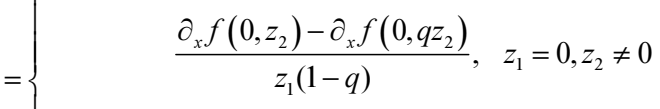

$$
\begin{aligned}
& \frac{\partial_{y} f\left(z_{1}, 0\right)-\partial_{y} f\left(q z_{1}, 0\right)}{z_{1}(1-q)}, z_{1} \neq 0, z_{2}=0 \\
& \partial_{y x} f(0,0) z_{1}=0, \quad z_{2}=0 \text {. }
\end{aligned}
$$

Now let $u=f\left(z_{1}, z_{2}\right)$, then:

$$
\begin{aligned}
& D_{q, x} f\left(z_{1}, z_{2}\right) D_{q, x x} f \\
& =\left\{\begin{array}{c}
\frac{\left(f\left(z_{1}, z_{2}\right)-f\left(q z_{1}, z_{2}\right)\right)\left(f\left(z_{1}, z_{2}\right)-2 f\left(q z_{1}, z_{2}\right)+f\left(q^{2} z_{1}, z_{2}\right)\right)}{z_{1}^{3}(1-q)^{3}}, z_{1} \neq 0 \\
\partial_{x} f\left(0, z_{2}\right)\left(\partial_{x x} f\left(0, z_{2}\right)\right), \quad z_{1}=0 .
\end{array}\right.
\end{aligned}
$$

Now substitute (3) and (4) in (2) we get the following case:

$$
\begin{aligned}
& \left(D_{q, x} u\right)\left(D_{q, x}^{2}\right)+\left(D_{q, y}^{2}\right) \\
& =\left\{\begin{array}{cc}
(3)+(4), & z_{1} \neq 0 \wedge z_{2} \neq 0 \\
\partial_{x} f\left(0, z_{2}\right)\left(\partial_{x x} f\left(0, z_{2}\right)\right)+\left.(3)\right|_{z_{1=0},} & z_{1}=0 \wedge z_{2} \neq 0 \\
\left.(4)\right|_{z_{2=0}}+\partial_{y y} f(0,0), & z_{1} \neq 0 \wedge z_{2}=0 \\
\left(\partial_{x} f(0,0)\right)\left(\partial_{x x} f(0,0)\right)+\partial_{y y} f(0,0), & z_{1}=0 \wedge z_{2}=0 .
\end{array}\right.
\end{aligned}
$$

The above discussion gives the following theorem.

\section{Theorem}

For $q \in(0,1)$, the solution of the transonic gas Equation 2 is given by:

$$
\begin{aligned}
& \sum_{i=1}^{r+1} \sum_{j=0}^{s-2}[i]_{q}[r-i+3]_{q}[r-i+2]_{q} \\
& a_{i, j} a_{r-i+3, s-j-2}=-[s]_{q}[s-1]_{q} a_{r, s} .
\end{aligned}
$$

\section{Proof}

Let q-transonic gas equation is $\left(D_{q, x} u\right)\left(D_{q, x}^{2}\right)+\left(D_{q, y}^{2}\right)=0$.

Then, from above discussions, we substitute $D_{q, x} u$, $D_{q, x}^{2}$ and $D_{q, y}^{2}$ in (2) we can find: 


$$
\begin{aligned}
& \frac{\left(f\left(z_{1}, z_{2}\right)-f\left(q z_{1}, z_{2}\right)\right)\left(f\left(z_{1}, z_{2}\right)-2 f\left(q z_{1}, z_{2}\right)+f\left(q^{2} z_{1}, z_{2}\right)\right)}{z_{1}^{3}(1-q)^{3}} \\
& +\frac{f\left(z_{1}, z_{2}\right)-2 f\left(z_{1}, q z_{2}\right)+f\left(z_{1}, q^{2} z_{2}\right)}{z_{2}^{2}-(1-q)^{2}}=0, z_{1}, z_{2} \neq 0 \\
& \frac{f^{2}\left(z_{1}, z_{2}\right)-3 f\left(z_{1}, z_{2}\right) f\left(q z_{1}, z_{2}\right)+f\left(z_{1}, z_{2}\right) f\left(q^{2} z_{1}, z_{2}\right)-2 f^{2}\left(q z_{1}, z_{2}\right)-f\left(q z_{1}, z_{2}\right) f\left(q^{2} z_{1}, z_{2}\right)}{z_{1}^{3}(1-q)^{3}} \\
& +\frac{f\left(z_{1}, z_{2}\right)-2 f\left(z_{1}, q z_{2}\right)+f\left(z_{1}, q^{2} z_{2}\right)}{z_{2}^{2}-(1-q)^{2}}=0
\end{aligned}
$$

Now let:

$$
u\left(z_{1}, z_{2}\right)=\sum_{n, m=0}^{\infty} a_{n, m} z_{1}^{n} z_{2}^{m}
$$

and:

$$
\begin{aligned}
& D_{q, x}\left(z_{1}^{n}\right)=\frac{z_{1}^{n}-\left(q z_{1}\right)^{n}}{z_{1}(1-q)} \\
& =\frac{z_{1}^{n}\left(1-q^{n}\right)}{z_{1}(1-q)} \\
& =z_{1}^{n-1}[n]_{q}
\end{aligned}
$$

This implies:

$$
\begin{aligned}
& \left(\sum_{n, m}^{\infty}[n]_{q} a_{n, m} z_{1}^{n-1} z_{2}^{m}\right)\left(\sum_{n, m}^{\infty}[n]_{q}[n-1]_{q} a_{n, m} z_{1}^{n-2} z_{2}^{m}\right) \\
& +\left(\sum_{n, m}^{\infty}[m]_{q}[m-1]_{q} a_{n, m} z_{1}^{n} z_{2}^{m-2}\right)=0 \\
& u\left(z_{1}, z_{2}\right)=\sum_{i, j, n, m}^{\infty}[i]_{q} a_{i, j} z_{1}^{i-1} z_{2}^{j}[n]_{q}[n-1]_{q} a_{n, m} z_{1}^{n-2} z_{2}^{m} \\
& +\sum_{n, m}^{\infty}[m]_{q}[m-1]_{q} a_{n, m} z_{1}^{n} z_{2}^{m-2}=0 \\
& \sum_{i, j, n, m}^{\infty}[i]_{q} a_{i, j} z_{1}^{i-1} z_{2}^{j}[n]_{q}[n-1]_{q} a_{n, m} z_{1}^{n-2} z_{2}^{m} \\
& +\sum_{n, m}^{\infty}[m]_{q}[m-1]_{q} a_{n, m} z_{1}^{n} z_{1}^{m-2}=0
\end{aligned}
$$

This implies:

$$
\begin{aligned}
& \sum_{i, j, n, m}^{\infty}\left([i]_{q}[n]_{q}[n-1]_{q} a_{i, j}\right) z_{1}^{i+n-3} z_{2}^{m+j} \\
= & \sum_{n, m}^{\infty}-[m]_{q}[m-1]_{q} a_{n, m} z_{1}^{n} z_{2}^{m-2}
\end{aligned}
$$

where:

$$
\begin{gathered}
\begin{array}{c}
m+j=s-2 \\
i+n-3=r
\end{array}
\end{gathered} \Rightarrow \begin{gathered}
m=s-j-2, m \geq 0 \Leftrightarrow s \geq j+2 \\
n=r-i+3, n \geq 2 \Leftrightarrow r-i+1 \geq 0 \Rightarrow r \geq i-1
\end{gathered}
$$

If and only if:

$$
\begin{aligned}
& \sum_{i=1, j=0 s=j+2, r=i-1}^{\infty}\left([i]_{q}[r-i+2]_{q}[r-i+2]_{q} a_{i, j} a_{r-i+3, s-j-2}\right) z_{1}^{r} z_{2}^{s-2} \\
= & \sum_{n, m=0}^{\infty}\left(-[m]_{q}[m-1]_{q} a_{n, m}\right) z_{1}^{n} z_{2}^{m-2}
\end{aligned}
$$$$
\sum_{r=0, s=2}^{\infty}\left(\sum_{i=1, j=1,}^{r+1} \sum_{j=2}^{s-2} \alpha_{i, r, s}\right) z_{1}^{r} z_{2}^{s-2}=\sum_{r=0, s=2}^{\infty}\left(-[s]_{q}[s-1]_{q} a_{r, s}\right) z_{1}^{r} z_{2}^{s-2},
$$

where:

$$
\alpha_{i, j, r, s}=\left([i]_{q}[r-i+2]_{q}[r-i+2]_{q} a_{i, j} a_{r-i+3, s-j-2}\right) .
$$

Hence, we deduce the following result:

$$
\begin{aligned}
& \sum_{i=1}^{r+1} \sum_{j=0}^{s-2}[i]_{q}[r-i+3]_{q}[r-i+2]_{q} \\
& a_{i, j} a_{r-i+3, s-j-2}=-[s]_{q}[s-1]_{q} a_{r, s} . \\
& \quad \forall r \geq 0 \text { and } s \geq 2, \forall r \in\{0,1,2, \ldots\} \text { and } s \in\{2,3,4, \ldots\}
\end{aligned}
$$

This conclude the proof.

\section{Conclusion}

In this study, the q-analogous of transonic gas equation presented. The classical solution considered. We show that the huge different between classical case and a new q-analogous of transonic gas equation.

In future I will investigate the solution of Equation (6) using code programming with the software Maple.

\section{Acknowledgement}

We would like to thank Dr. H. Rguigui for his help and guidance in this paper. Also thank anonymous referees for their careful reading of our manuscript and helpful suggestions.

\section{Ethics}

The author declare that there is no conflict interests regarding the publication of this manuscript. This article is original and contains unpublished material. 


\section{References}

Abdi, W.H., 1962. On certain q-difference equations and q-Laplace transforms, Proc. Nat. Inst. Sci. India Acad., 28: 1-15.

Accardi, L. and A. Boukas, 2007. Lie algebras associated with the renormalized higher powers of white noise. Commun. Stoch. Anal., 1: 57-69.

Accardi, L. and A. Boukas, 2009. Quantum probability, renormalization and infinite-dimensional *-lie algebras, Symmetry Integrability Geometry: Meth. Applic., 5: 1-31.

Accardi, L. and A. Boukas, 2006. Renormalized Higher Powers of White Noise (RHPWN) and conformal field theory. Infin. Dimens. Anal. Quantum Probab. Relat. Top., 9: 353. DOI: $10.1142 / \mathrm{S} 021902570600241 \mathrm{X}$

Accardi, L., A. Boukas and U. Franz, 2006. Renormalized powers of quantum white noise. Infin. Dimens. Anal. Quantum. Probab. Relat. Top., 9: 129. DOI: $10.1142 / \mathrm{S} 0219025706002263$

Adams, C.R., 1929. On the linear ordinary q-difference equation, Annal. Math., 30: 195-205. DOI: $10.2307 / 1968274$

Altoum, S.H., H.A. Othman and H. Rguigui, 2017. Quantum white noise Gaussian kernel operators. Chaos, Solitons Fractals, 104: 468-476. DOI: $10.1016 /$ j.chaos.2017.08.039

Bangerezako, G., 2004. Variational q-calculus. J. Math. Anal. Applic., 289: 650-665.

DOI: 10.1016/j.jmaa.2003.09.004

Bożejko, M. and R. Speicher, 1991. An example of a generalized Brownian motion. Commun. Math. Phys., 137: 519-531. DOI: 10.1007/BF02100275
Ettaieb, A., H. Ouerdiane and H. Rguigui, 2012. Cauchy problem and integral representation associated to the power of the QWN-Euler operator. Commun. Stochastic Anal., 6: 615-627.

Ettaieb, A., H. Ouerdiane and H. Rguigui, 2014. Higher powers of quantum white noise derivatives. Commun. Stochastic Anal., 8: 531-550.

Ettaieb, A., N.T. Khalifa, H. Ouerdiane and H. Rguigui, 2016. Higher powers of analytical operators and associated *-Lie algebras. Infin. Dimens. Anal. Quantum. Probab. Relat. Top., 19: 20. DOI: $10.1142 / \mathrm{S} 0219025716500132$

Hida, T., 1993. A Role of the Lévy Laplacian in the Causal Calculus of Generalized White Noise Functionals. In: Stochastic Processes, a Festschrift in Honour, Kallianpur, G. and S. Combines (Ed.), Springer-Verlag.

Jackson, H.F., 1910. Q-Difference equations. Am. J. Math., 32: 305-314. DOI: 10.2307/2370183

Leeuwen, H.V. and H. Maassen, 1995. A q-deformation of the Gauss distribution. J. Math. Phys., 36: 4743-4756.

Mason, T.E., 1915. On properties of the solutions of linear q-difference equations with entire function coefficients. Am. J. Math., 37: 439-444. DOI: $10.2307 / 2370216$

Titov, S.S., 1988. A Method of Finite-Dimensional Rings for Solving Nonlinear Equations of Mathematical Physics. In: Aerodynamics, Ivanova, T.P. (Ed.), Saratov Univ., Saratov, pp: 104-110.

Yuri, G.K., J.L. da Silva, L. Streit and F.U. Georgi, 1998. Analysis on Poisson and Gamma spaces. Infinite dimensional anal. Quant. Probab. Rel. Top., 1: 91-91. DOI: 10.1142/S0219025798000089 